\title{
Correction to: Theoretical investigation on the water-assisted excited-state proton transfer of 7-azaindole derivatives: substituent effect
}

Jiacheng $\mathbf{Y i}^{1} \cdot$ Hua Fang ${ }^{1}$

Published online: 3 November 2017

(C) Springer-Verlag GmbH Germany 2017

Correction to: J Mol Model (2017) 23:312

https://doi.org/10.1007/s00894-017-3487-5

The original version of this article unfortunately contained a mistake in Table 3. This correct Table 3 is given below.

Table 3 Reaction energies $(\Delta E)$ and barrier heights $(\Delta V)$ for excitedstate proton transfer in $2 \mathrm{RAI}-\mathrm{H}_{2} \mathrm{O}\left(\mathrm{R}=\mathrm{H}, \mathrm{OH}, \mathrm{OCH}_{3} ; \mathrm{CN}, \mathrm{CHO}, \mathrm{NO}_{2}\right)$ complexes in water

\begin{tabular}{llr}
\hline Complex & $\Delta V$ & \multicolumn{1}{l}{$\Delta E$} \\
\hline $7 \mathrm{AI}-\mathrm{H}_{2} \mathrm{O}^{\mathrm{a}}$ & $10.6(6.86)$ & $-13.6(-13.2)$ \\
$2(\mathrm{OH}) \mathrm{AI}-\mathrm{H}_{2} \mathrm{O}$ & $9.43(6.20)$ & $-15.9(-15.4)$ \\
$2\left(\mathrm{OCH}_{3}\right) \mathrm{AI}-\mathrm{H}_{2} \mathrm{O}$ & $9.38(5.86)$ & $-15.4(-14.8)$ \\
$2\left(\mathrm{NO}_{2}\right) \mathrm{AI}-\mathrm{H}_{2} \mathrm{O}$ & $15.6(12.8)$ & $0.10(-0.10)$ \\
$2(\mathrm{CN}) \mathrm{AI}-\mathrm{H}_{2} \mathrm{O}$ & $10.2(7.30)$ & $-13.4(-13.1)$ \\
$2(\mathrm{CHO}) \mathrm{AI}-\mathrm{H}_{2} \mathrm{O}$ & $19.7(15.6)$ & $-2.90(-2.70)$ \\
\hline
\end{tabular}

The numbers in parentheses include zero-point energies. Energies are in $\mathrm{kcal} / \mathrm{mol}$.

${ }^{\mathrm{a}}$ Data from Ref [1]

The online version of the original article can be found at https://doi.org/ $10.1007 / \mathrm{s} 00894-017-3487-5$

\section{Hua Fang}

susanfang20@gmail.com

1 Department of Chemistry and Material Science, College of Science, Nanjing Forestry University, Nanjing 210037, People's Republic of China

\section{Reference}

1. Yi J, Fang H (2017). Photochem. Photobiol. https://doi.org/10.1111/ php.12839 